\title{
Different Quantization Mechanisms in Single-Electron Pumps Driven by Surface Acoustic Waves
}

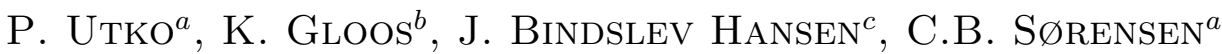 \\ AND P.E. LINDELOF ${ }^{a}$ \\ ${ }^{a}$ Nano-Science Center, Niels Bohr Institute, University of Copenhagen \\ Universitetsparken 5, 2100 Copenhagen, Denmark \\ ${ }^{b}$ Wihuri Physical Laboratory, Department of Physics \\ University of Turku, 20014 Turku, Finland \\ ${ }^{c}$ Department of Physics, Technical University of Denmark \\ 2800 Lyngby, Denmark
}

We have studied the acoustoelectric current in single-electron pumps driven by surface acoustic waves. We have found that in certain parameter ranges two different sets of quantized steps dominate the acoustoelectric current versus gate-voltage characteristics. In some cases, both types of quantized steps appear simultaneously though at different current values, as if they were superposed on each other. This could indicate two independent quantization mechanisms for the acoustoelectric current.

PACS numbers: 73.23.-b, 72.50.+b, 73.21.La

\section{Introduction}

During the last two decades single-electron devices have attracted a lot of interest. In one class of such systems, surface acoustic waves (SAWs) are used to transfer a discrete number of electrons across a quantum point contact (QPC) operated in the closed-channel regime [1-6]. This is often described using a model of "moving quantum dots" [1], where each dot, formed by the dynamic SAW potential and the static barrier of the QPC, captures electrons at the QPC entrance. Coulomb repulsion between the trapped electrons restricts their number inside the dot. As a result, the SAW-induced acoustoelectric current develops plateaus at $I=n e f$, where $n$ is an integer, $e$ - the electron charge, and $f$ - the SAW frequency. The current quantization can be observed as a function of the gate and bias voltages, the SAW power, and frequency. 
Here we have investigated the response of the acoustoelectric current to changes in the gate voltage $V_{\mathrm{g}}$ and the SAW frequency $f$. Within a certain frequency range, two separate sets of plateaus seemed to dominate the $I\left(V_{\mathrm{g}}\right)$ characteristics. In some cases, both types of quantized steps appeared simultaneously, though at different current values, as if they were superposed on each other. Their presence could result from two independent quantization mechanisms for the acoustoelectric current.

\section{Experiment}

The inset to Fig. 1a shows a schematic layout of our devices. Two aluminum interdigital transducers (IDTs) can be used to generate the SAW. They are deposited $2.6 \mathrm{~mm}$ apart, on both sides of a 2DEG mesa with a QPC in the center. The IDT electrode spacing sets the fundamental wavelength and frequency of the SAW to about $1.15 \mu \mathrm{m}$ and $2.45 \mathrm{GHz}$, respectively. The GaAs/AlGaAs heterostructure

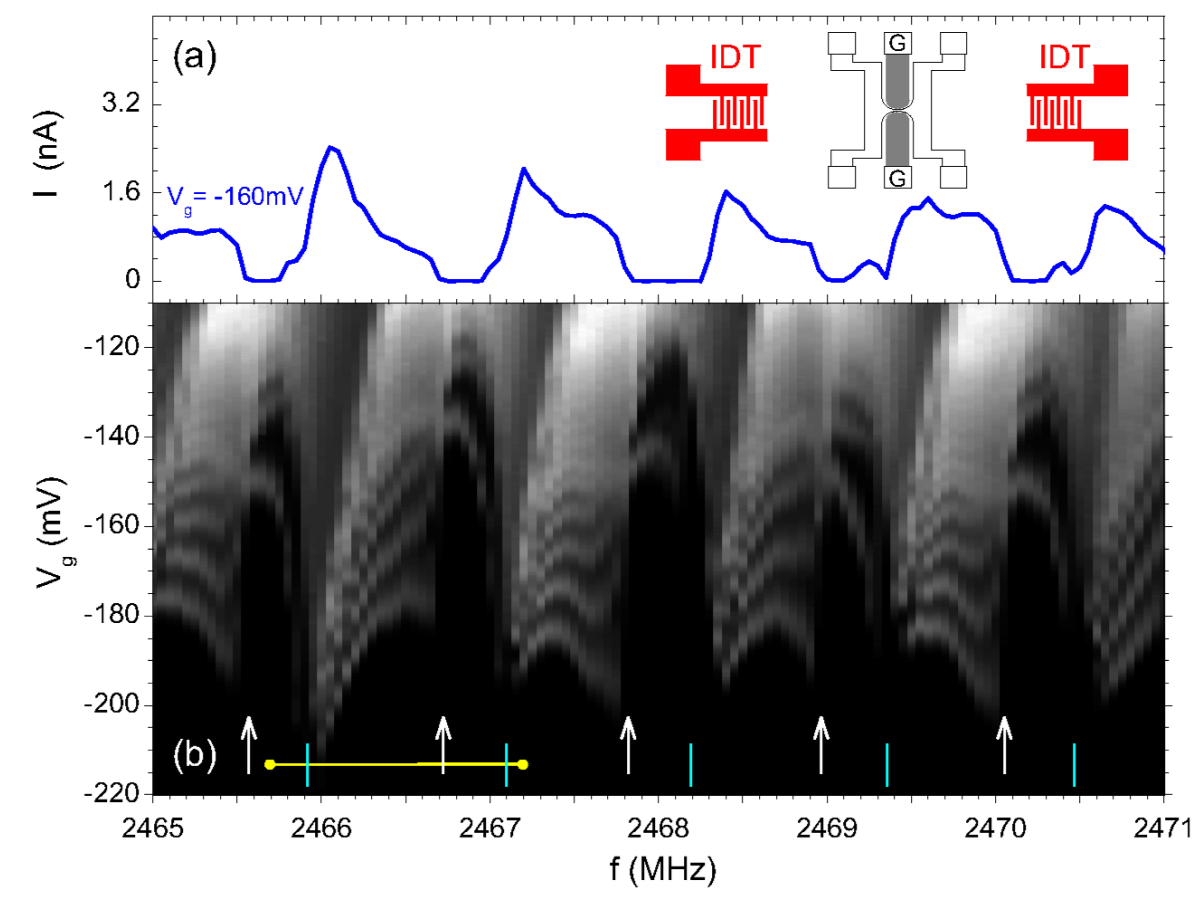

Fig. 1. (a) Acoustoelectric current $I$ as a function of the SAW frequency $f$. Inset shows a schematic layout of the device. (b) Gray-scale coded plot of transconductance $\mathrm{d} I / \mathrm{d} V_{\mathrm{g}}$ with respect to both the SAW frequency $f$ and gate voltage $V_{\mathrm{g}}$. Dark (light) indicates small (large) values of the current derivative. The vertical arrows and bars mark the frequencies around which one set of acoustoelectric plateaus is replaced by another one, see the text for details. The horizontal line indicates the frequency range from which the traces in Fig. 2 are selected. All measurements were taken at $P=+9.8 \mathrm{dBm}$ and $T=1.8 \mathrm{~K}$. 
has a mobility of $105 \mathrm{~m}^{2} /(\mathrm{V} \mathrm{s})$ and a carrier density of $2.8 \times 10^{15} \mathrm{~m}^{-2}$, measured in the dark at $10 \mathrm{~K}$. The QPC is patterned by electron-beam lithography. Two semicircular trenches are shallow-etched to form a smooth constriction between two electron reservoirs, whereas large areas of the 2DEG across the channel serve as side gates. Depending on the device, the trenches have a curvature radius of 5.0, 7.5 or $10.0 \mu \mathrm{m}$. They are $200 \mathrm{~nm}$ wide and $40 \mathrm{~nm}$ deep. See Refs. [5, 6] for more details on the device layout.

The rf excitation of power $P$ could be applied to one of the two IDTs, or split up and simultaneously fed to both transducers. With a phase shifter and an attenuator in one of the $\mathrm{rf}$ lines, the relative magnitude and the relative phase of both signals could then be varied and adjusted. A low-noise current preamplifier detected the acoustoelectric current. The samples were investigated either in a ${ }^{3} \mathrm{He}$ refrigerator operating at $1.2 \mathrm{~K}$ or a ${ }^{4} \mathrm{He}$ refrigerator operating at $1.8 \mathrm{~K}$. The lower base temperature of the ${ }^{3} \mathrm{He}$ cryostat did not help to improve the results because in both systems the 2DEG of our devices was heated up to around $5 \mathrm{~K}$ at the typically applied $\mathrm{rf}$ powers of around 10-15 $\mathrm{dBm}[7]$.

Figures $1 \mathrm{a}$ and $2 \mathrm{a}$ show typical $I(f)$ and $I\left(V_{\mathrm{g}}\right)$ characteristics, respectively, obtained when only one IDT was used to generate the SAW. The pronounced 1.1 $\mathrm{MHz}$ beat period of the current indicates that the interference of the surface

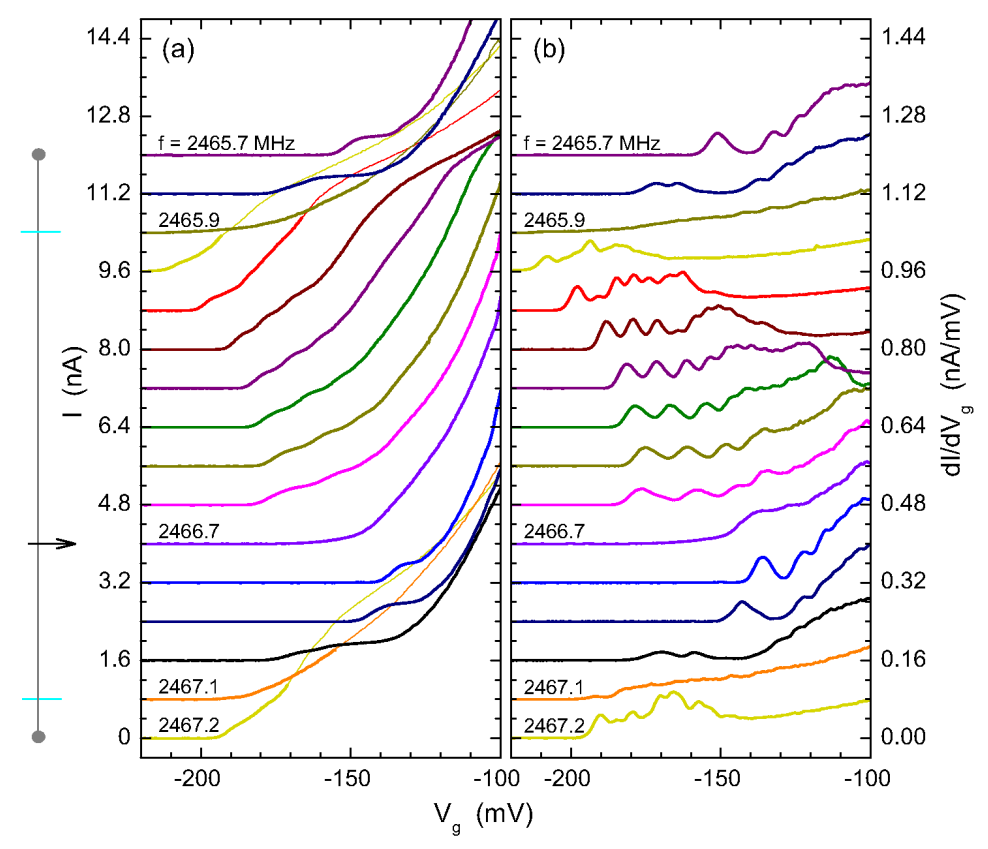

Fig. 2. (a) Acoustoelectric current $I$ and (b) transconductance $\mathrm{d} I / \mathrm{d} V_{\mathrm{g}}$ as a function of gate voltage $V_{\mathrm{g}}$. The 16 current (transconductance) traces were recorded at fixed SAW frequencies from 2465.7 to $2467.2 \mathrm{MHz}$, in steps of $0.1 \mathrm{MHz}$. The curves were successively offset in the vertical direction by $0.8 \mathrm{nA}(0.08 \mathrm{nA} / \mathrm{mV})$. The measurements were carried out at $P=+9.8 \mathrm{dBm}$, and $T=1.8 \mathrm{~K}$. 
acoustic wave with reflected waves matters. This is supported by the results obtained when a second, independent SAW beam was added, traveling in opposite direction [8]. Current plateaus at integer multiples of ef can be resolved in either $I(f)$ or $I\left(V_{\mathrm{g}}\right)$ traces. However, they become more apparent in the current derivative, see Figs. $1 \mathrm{~b}$ and $2 \mathrm{~b}$. Thus, to enhance the resolution of the acoustoelectric transitions, we have routinely monitored the transconductance $\mathrm{d} I / \mathrm{d} V_{\mathrm{g}}$ along with the current $I$. This was done by adding a small $117 \mathrm{~Hz}$ modulation of $\mathrm{d} V_{\mathrm{g}} \approx 0.5 \mathrm{mV}$ to the gate voltage, and measuring the ac component of the current with a lock-in amplifier.

The gray-scale plot of $\mathrm{d} I / \mathrm{d} V_{\mathrm{g}}$ in Fig. $1 \mathrm{~b}$ reveals a periodic structure with respect to frequency, with a beat period of roughly $1.1 \mathrm{MHz}$. However, transconductance minima corresponding to current plateaus do not evolve smoothly over the entire $1.1 \mathrm{MHz}$ period. On increasing the SAW frequency, broad plateaus with respect to $V_{\mathrm{g}}$ are abruptly replaced by densely-packed quantized steps. Frequencies around which such transitions occur are marked with vertical bars. Arrows indicate another type of transition where the acoustoelectric current drastically shifts its onset along the $V_{\mathrm{g}}$-axis in response to a small change in frequency.

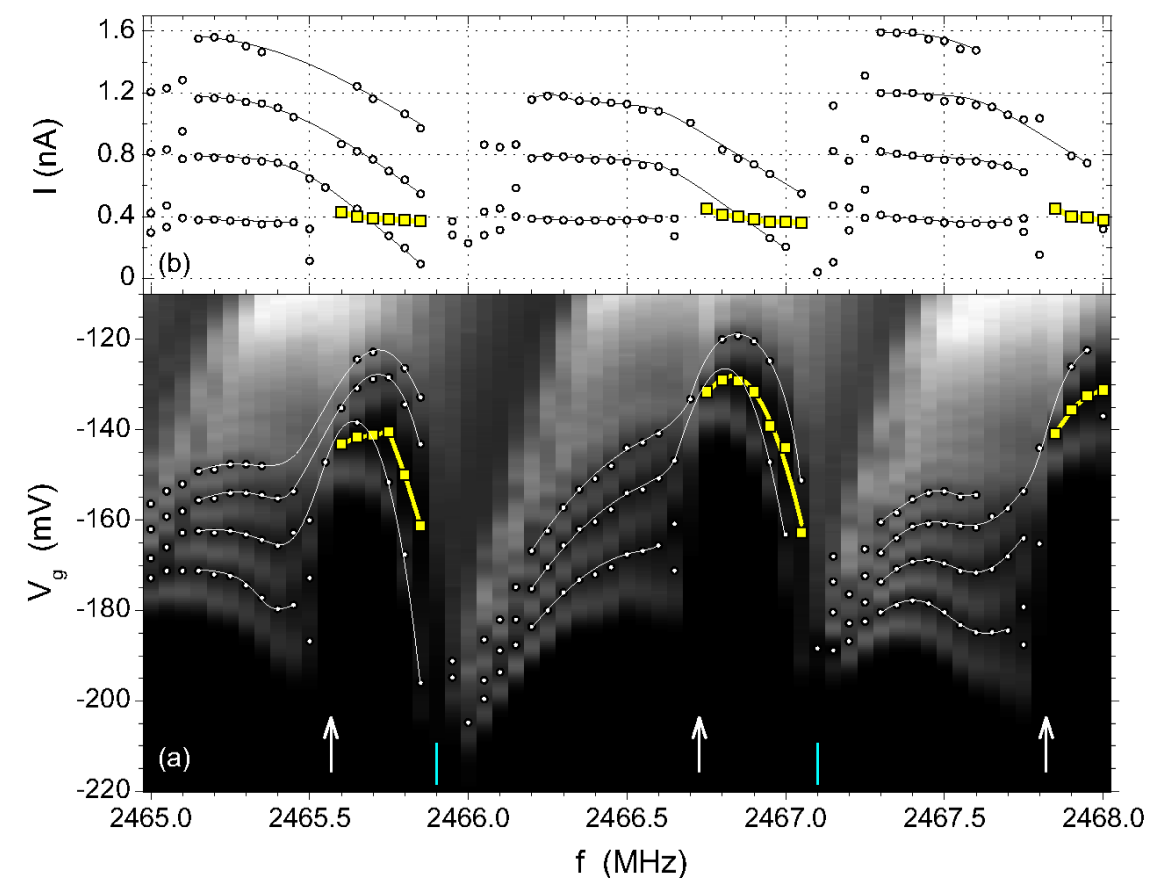

Fig. 3. (a) Gray-scale plot of transconductance $\mathrm{d} I / \mathrm{d} V_{\mathrm{g}}$ with respect to SAW frequency $f$ and gate voltage $V_{\mathrm{g}}$. Open circles and full squares mark the position of transconductance minima. (b) Acoustoelectric current $I$ at the minima indicated in (a). In both (a) and (b), two different kinds of acoustoelectric plateaus can be distinguished. Solid lines through the data points are guides to the eye. The measurements were carried out at $P=+9.8 \mathrm{dBm}$ and $T=1.8 \mathrm{~K}$. 
Those features become more apparent in Fig. 2, showing selected $I\left(V_{\mathrm{g}}\right)$ and $\mathrm{d} I\left(V_{\mathrm{g}}\right) / \mathrm{d} V_{\mathrm{g}}$ traces taken at frequencies from 2465.7 to $2467.2 \mathrm{MHz}$. In both Fig. 1b and 2, two frequency intervals can be distinguished within a beat period of $\sim 1.1 \mathrm{MHz}$ where two different sets of plateaus dominate the $I\left(V_{\mathrm{g}}\right)$ characteristics. Those two sets seem to replace each other around certain frequencies, indicated by arrows and bars. However, in some cases both sets can also appear simultaneously in the $I\left(V_{\mathrm{g}}\right)$ traces, as if they were superposed onto each other. The dominating set consists then of broad current plateaus with respect to the gate voltage that are well-defined at the expected multiples of $e f$. On the other hand, weakly-pronounced steps belonging to the second set are formed below those ideal values, as highlighted in Fig. 3. Both sets respond differently to the SAW frequency, as for example in the range from 2465.7 to $2465.9 \mathrm{MHz}$ in Fig. 3. When the frequency is incremented within such a range, one plateau in $I\left(V_{\mathrm{g}}\right)$ remains close to the expected value of $e f \approx 400 \mathrm{pA}$, while the other set of quantized steps appears at lower and lower currents.

\section{Discussion and conclusions}

The frequency response measurements presented here reveal a complicated pattern of the acoustoelectric transitions. Their most apparent feature, the $1.1 \mathrm{MHz}$ beating of the acoustoelectric current, immediately reminds us of the interference patterns due to a standing wave. In fact, the results obtained while varying the SAW frequency closely resemble those when the phase is controlled between two counter-propagating SAW beams [8]. The period of $1.1 \mathrm{MHz}$ thus corresponds to a phase shift of $2 \pi$. This already indicates that the standing wave matters, but it does not specify where to find its nodes.

The SAW-driven single-electron transport is usually described using a model of moving quantum dots [1]. In such a model, single electrons are trapped in the SAW minima and transferred across the QPC barrier, which is assumed to be long with respect to the SAW wavelength. As the dot moves towards the center of the constriction and its size decreases, the Coulomb repulsion between the trapped electrons restricts their number inside the dot, forcing some of them to escape back to the 2DEG reservoir they were captured from. Thus, the minimum size of the dot determines the final number $n$ of transferred electrons.

In this [1] and other theoretical models [9, 10], the static barrier of the QPC is assumed to be long with respect to the SAW wavelength. However, we believe that this is not entirely valid for our devices. In spite of large nominal lengths of our QPCs $(\sim 2 \mu \mathrm{m})$, their properties are determined by rather short barriers of around $0.2 \mu \mathrm{m}[11]$, that is about $1 / 5$ of the SAW wavelength. Such a short barrier within the QPC channel could reduce the number of electrons that are further carried in a moving quantum dot. We emphasize the main difference with respect to the models relying solely on long QPC barriers $[1,9,10]$ where only the low-energy electrons at the bottom of the dot are transferred across the constriction. This is no longer the case if the moving quantum dot approaches a short (though 
high) barrier. Electrons in the lowest energy states of the SAW minimum are then held back at the barrier and return to the reservoir they originated from. Only those with higher energy are transferred across the QPC and contribute to the acoustoelectric current.

Those short potential barriers in our nominally long constrictions could lead to an additional quantization mechanism, independent from that described by the standard model of "moving quantum dots". Expelling of low-energy electrons at the short static barrier can take place in series with the quantization mechanisms described in previous models which rely on the escape or capture of high-energy electrons $[1,9,10]$. The already quantized current can thus be further reduced at the short barrier, also to a new quantized level. This could explain Fig. 3: The higher order plateaus (thin lines/points) are lowered when the second mechanism (thick line/points) sets in. Thus, this simple model explains the presence of at least two different kinds of current plateaus. One could further speculate that, at very narrow channels, more than one barrier is present in the constriction. One set of acoustoelectric plateaus could then result from operating the device as a static quantum dot with two separate barriers of comparable magnitude, as found in Ref. [4].

\section{Acknowledgments}

This work was supported by the European Commission FET Project SAWPHOTON. P.U. acknowledges support from EC FP6 funding (contract No. FP6-2004-IST-003673).

\section{References}

[1] J.M. Shilton, V.I. Talyanskii, M. Pepper, D.A. Ritchie, J.A. Frost, C.J.B. Ford, C.G. Smith, G.A.C. Jones, J. Phys., Condens. Matter 8, L531 (1996).

[2] J. Cunningham, V.I. Talyanskii, J.M. Shilton, M. Pepper, A. Kristensen, P.E. Lindelof, Phys. Rev. B 62, 1564 (2000).

[3] J. Ebbecke, G. Bastian, M. Blcker, K. Pierz, F.J. Ahlers, Appl. Phys. Lett. 77, 2601 (2000).

[4] N.E. Fletcher, J. Ebbecke, T.J.B.M. Janssen, F.J. Ahlers, M. Pepper, H.E. Beere, D.A. Ritchie, Phys. Rev. B 68, 245310 (2003).

[5] P. Utko, K. Gloos, J. Bindslev Hansen, P.E. Lindelof, Acta Phys. Pol. A 103, 533 (2003).

[6] K. Gloos, P. Utko, J. Bindslev Hansen, P.E. Lindelof, Phys. Rev. B 70, 235345 (2004).

[7] P. Utko, P.E. Lindelof, K. Gloos, Appl. Phys. Lett. 88, 202113 (2006).

[8] P. Utko, J. Bindslev Hansen, P.E. Lindelof, C.B. Sørensen, K. Gloos, to be published in J. Low Temp. Phys.

[9] K. Flensberg, Q. Niu, M. Pustilnik, Phys. Rev. B 60, R16 291 (1999).

[10] A.M. Robinson, C.H.W. Barnes, Phys. Rev. B 63, 165418 (2001).

[11] K. Gloos, P. Utko, M. Aagesen, C.B. Sørensen, J. Bindslev Hansen, P.E. Lindelof, Phys. Rev. B 73, 125326 (2006). 